\title{
Die unsichtbare Hand der Ampel: sozialökologische Marktwirtschaft
}

Mit der neuen Bundesregierung ist frischer Wind ins politische Berlin eingekehrt (Schnitzer, 2021). Noch während der Koalitionsverhandlungen wurde vor einem Scheitern gewarnt. Umso überraschender, dass der Koalitionsvertrag einige Brücken auf den Gebieten der Sozial-, Klima- und Finanzpolitik schlägt. Dort heißt es: „Es gilt, die soziale Marktwirtschaft als eine sozial-ökologische Marktwirtschaft neu zu begründen." Vielleicht hat im Vorfeld die Fantasie dafür gefehlt, dass Wirtschaftspolitik nicht nur aus Links und Rechts besteht, nicht nur aus Markt oder Staat, sondern dass unser Wirtschaftssystem eine „gemischte Wirtschaft" ist, in dem wohltemperierte Staatseingriffe für das Funktionieren der Marktwirtschaft nötig sind und "die richtige Balance zwischen dem öffentlichen und privaten Sektor“ (Stiglitz, 1999, 16) gesucht wird. Auch der Begriff der „Sozialen Marktwirtschaft" ist verschiedenen Interpretationen zugänglich und über die korrekte Verwendung wird trefflich gestritten. Aktivistischere Ansätze, wie sie vom früheren Bundeswirtschaftsminister Karl Schiller (1971) verfolgt wurden, mussten sich gegen Kritik zur Wehr setzen, die Soziale Marktwirtschaft mit „Dirigismus“ zu bedrohen (Erhard, 1971). Einem ähnlichen Vorwurf sieht sich der neue Aktivismus unter anderem in der Klimapolitik ausgesetzt (Fuest, 2020). Sinnvollerweise setzt die neue Koalition gerade beim Abbau von Dirigismus an (Bürgergeldkonzept, Verfahrensbeschleunigungen beim Ausbau erneuerbarer Energien).

Teilt man die Wirtschaftspolitik in die drei Funktionen der Staatstätigkeit (Allokation, Verteilung, Stabilisierung) nach Musgrave (1959), so widmet sich jede Koalitionspartei einem der Themen inhaltlich in besonderer Weise und besetzt die jeweiligen Schlüsselressorts.

Mit der Klimapolitik hat die Ampel ein allokationspolitisches Thema par excellence ins Zentrum gerückt. In der Theorie scheint die Aufgabe leicht: Ist eine Verschmutzung zulasten der Gesellschaft identifiziert (negativer externer Effekt), wäre den Verschmutzenden eine (Pigou-)Steuer aufzuerlegen, die den Schaden internalisiert und eine entsprechende Lenkungswirkung entfaltet. In der Praxis kollidiert eine angemessen hohe $\mathrm{CO}_{2}$-Abgabe jedoch mit anderen Zielen. Die energieintensive Industrie würde belastet (Stabilisierung) sowie Verbrauchende mit geringen Einkommen, die einen hohen Einkommensanteil für Energie aufwenden (Verteilung). Zudem ist die Nachfrage nach verschmutzungsintensivem Konsum kurzfristig unelastisch, was die Lenkungswirkung des $\mathrm{CO}_{2}$-Preises beeinträchtigt. Der sich an höheren indirekten Abgaben entzündbare Protest ist nicht zu unterschätzen, was z.B. die Gelbwestenbewegung in Frankreich gezeigt hat. Das alleinige Setzen auf den $\mathrm{CO}_{2}$-Preis könnte sich daher als ebenso politisch suizidal erweisen wie die rein gesinnungsethisch motivierte Nutzung von Verboten und Dirigismus, weshalb zunächst die Voraussetzungen für elastische Nachfrage und marktbasierte Lösungen geschaffen werden müssen, was Substitutionsmöglichkeiten voraussetzt. Derzeit fehlt es an Substituten, wie günstigen Elektroautos, erneuerbarer Energie und öffentlichen Verkehrsangeboten.

Die Herausforderung des neuen Wirtschafts- und Klimaministeriums besteht darin, einen Rahmen dafür bereitzustellen, dass die Substitute des fossilen Verbrauchs so marktkonform und kosteneffizient wie möglich produziert werden können, z.B. mit Verfahrensbeschleunigung, aber auch durch ein Strommarktdesign, das saubere Energien durch Entlastungen bzw. relative Subventionierung fördert. Als Zielmarke für den Ausbau der Erneuerbaren wird 2030 angegeben. Bis dahin sollen $80 \%$ des Stromverbrauchs durch erneuerbare Energien und $50 \%$ der Wärme klimaneutral erzeugt werden. 200 Gigawatt (GW) Strom

(c) Der/die Autor:in 2022. Open Access: Dieser Artikel wird unter der Creative Commons Namensnennung 4.0 International Lizenz veröffentlicht (creativecommons.org/licenses/by/4.0/deed.de).

Open Access wird durch die ZBW - Leibniz-Informationszentrum Wirtschaft gefördert.

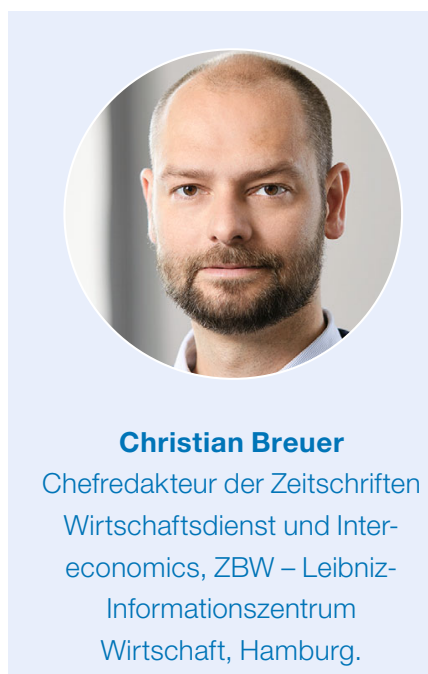

Wirtschaft, Hamburg. 
sollen durch Photovoltaik und mindestens $30 \mathrm{GW}$ durch Offshore-Windenergie erzeugt werden. Das Ausbautempo sei zu verdreifachen. Zudem sollen 2030 der Kohleausstieg vollzogen, 15 Mio. vollelektrische Pkw zugelassen sowie 1 Mio. Ladepunkte zugänglich sein. Anstatt die höheren Energiepreise als „Klimaprämie“ an die Verbrauchenden zurückzugeben, wird ab 2023 die EEG-Umlage abgeschafft, was den Strompreis senkt und Anreize für die Verstromung setzt. Noch konsequenter wäre, die Stromsteuer ebenfalls abzuschaffen und lediglich Energie aus Kohle und Gas mit einer Pigou-Steuer zu belasten. Dies würde saubere Energie entlasten und so den Markt zum Zubau bei Erneuerbaren drängen.

Verteilungspolitische Maßnahmen werden in der Lohn- und Sozialpolitik angekündigt. Der Mindestlohn steigt (voraussichtlich noch 2022) auf 12 Euro und die Mini- und Midijobs sollen erweitert werden, von 450 auf 520 bzw. 1.300 auf 1.600 Euro. Hartz IV soll durch ein Bürgergeld ersetzt werden, dessen Ausgestaltung noch zu konkretisieren ist. Eine einheitliche Kindergrundsicherung soll die Kakofonie in der Sozialpolitik für Familien beenden, was einer Entlastung für Alleinerziehende und Familien mit kleinen und mittleren Einkommen entspricht (Breuer, 2018). Die Kombination aus höherem Mindestlohn und Entlastung von Geringverdienenden dürfte das Nettoeinkommen im Niedriglohnbereich erhöhen und die „Armutsfallen“ im Zusammenhang mit den Leistungen des SGB II entschärfen. Dies würde niedrigere Transferentzugsraten ermöglichen, was die Bürokratie (bzw. den Dirigismus) der Anrechnungen und Sanktionen teilweise entbehrlich machen könnte (Breuer, 2019).

Stabilisierungspolitisch verpflichtet sich die Ampel zunächst auf die Rückkehr zur Schuldenbremse 2023. Obwohl dies umstritten ist (Stiglitz und Tooze, 2021), war immer bekannt, dass die Schuldenbremse im Grundgesetz nicht mit der einfachen Mehrheit der Ampelkoalition im Bundestag zu ändern ist und insofern das Festhalten an ihr schlicht die normative Kraft des Faktischen. Vielmehr geht es darum, die Schuldenbremse zu „interpretieren, anstatt sie zu verändern“. Will eine Regierung die Finanzpolitik expansiver ausrichten, kann sie dies auch mit der Schuldenbremse tun (Breuer, 2021), z.B. indem explizite durch implizite Verschuldung substituiert wird (etwa durch Renten- oder Subventionsversprechen in der Zukunft, was z. B. mit dem neuen EEG geschehen könnte). Außerdem könnten Zahlungen außerhalb des regulären Budgets verbucht oder die Ausnahmeklausel bzw. die Wiedereinführung der Schuldenbremse kreativ ausgelegt werden (Boysen-Hogrefe et al., 2022). Auch Letzteres wäre nicht völlig neu (Hulverscheidt, 2010). Zudem wird eine Reform des Stabilitäts- und Wachstumspakts auf EUEbene angekündigt, in der sich die Regierung an den Zielen Wachstum, Investitionen, Einfachheit und Transparenz orientieren möchte. Andere EU-Länder setzen ebenfalls Signale in Richtung einer stärkeren Investitionsorientierung (Draghi und Macron, 2021).

Für die Symbolik des Bundesfinanzministers sind sowohl die verteilungs- als auch die klimapolitischen Maßnahmen ein Geschenk: Beides stimuliert die Binnenkonjunktur, ohne dass Defizite im Kernhaushalt benötigt werden. Mit den damit induzierten Steuereinnahmen konsolidiert sich der Bundeshaushalt wie durch eine „unsichtbare Hand“.

\section{Literatur}

Boysen-Hogrefe, J. et al. (2022), Haushaltspolitik der neuen Bundesregierung, Wirtschaftsdienst, 102(1), 6-26. Breuer, C. (2018), Ein Grundeinkommen für Kinder, Wirtschaftsdienst, 98(7), 481-488.

Breuer, C. (2019), Dilemma Hartz IV: Geringverdiener entlasten, Wirtschaftsdienst, 99(2), 82-83.

Breuer, C. (2021), Nützt die Schuldenbremse den kommenden Generationen?, Wirtschaftsdienst, 101(7), 490-491. Draghi, M. und E. Macron (2021), The EU's fiscal rules must be reformed, Financial Times, 23. Dezember. Erhard, L. (1971), Haben wir noch eine Soziale Marktwirtschaft?, Wirtschaftsdienst, 51 (12) 627-631.

Fuest, C. (2020), Die schleichende Verbreitung des Neodirigismus, Frankfurter Allgemeine Zeitung, 7. Februar. Hulverscheidt, C. (2010), Die Tricksereien des Finanzministers, Süddeutsche Zeitung, 25. Oktober.

Musgrave, R. A. (1959), The Theory of Public Finance - A Study in Public Economy, McGraw-Hill. 